\title{
Pharmacodynamic Effects, Pharmacokinetics, and Metabolism of the Synthetic Cannabinoid AM-2201 in Male Rats
}

\author{
Jeremy Carlier, ${ }^{1}$ Ariane Wohlfarth, ${ }^{2}$ Bonita D. Salmeron, Karl B. Scheidweiler, \\ Marilyn A. Huestis, ${ }^{3}$ and Michael H. Baumann \\ Chemistry and Drug Metabolism (J.C., A.W., K.B.S., M.A.H.) and Designer Drug Research Unit (B.D.S., M.H.B.), Intramural \\ Research Program, National Institute on Drug Abuse (NIDA), National Institutes of Health (NIH), Baltimore, Maryland
}

Received May 8, 2018; accepted September 6, 2018

\begin{abstract}
Novel synthetic cannabinoids are appearing in recreational drug markets worldwide. Pharmacological characterization of these new drugs is needed to inform clinicians, toxicologists, and policy makers who monitor public health. [1-(5-Fluoropentyl)-1H-indol-3-yl](1-naphthyl)methanone (AM-2201) is an abused synthetic cannabinoid that was initially created as a research tool for investigating the endocannabinoid system. Here we measured the pharmacodynamic effects of AM-2201 in rats, and simultaneously determined plasma pharmacokinetics for the parent drug and its metabolites. Male SpragueDawley rats were fitted with surgically implanted temperature transponders and indwelling jugular catheters under pentobarbital anesthesia. One week later, rats received subcutaneous injection of AM-2201 $(0.1,0.3$, and $1.0 \mathrm{mg} / \mathrm{kg})$ or its vehicle, and serial blood specimens were withdrawn via catheters. Core temperatures and catalepsy were measured just prior to each blood withdrawal, and plasma was assayed for drug and
\end{abstract}

metabolites using liquid chromatography-tandem mass spectrometry. We found that AM-2201 produced dose-related hypothermia and catalepsy that peaked at 2 hours and lasted up to 8 hours. AM-2201 plasma concentrations rose linearly with increasing dose and ranged from 0.14 to $67.9 \mu \mathrm{g} / \mathrm{l}$. Concentrations of three metabolites, AM-2201 N-(4-hydroxypentyl) ( $\leq 0.17 \mu \mathrm{g} / \mathrm{l})$, naphthalen-1-yl-(1-pentylindol-3-yl)methanone (JWH-018) $N$-(5-hydroxypentyl) $(\leq 1.14 \mu \mathrm{g} / \mathrm{l})$, and JWH-018 $N$-pentanoic acid $(\leq 0.88 \mu \mathrm{g} / \mathrm{l})$ were detectable but much lower. Peak AM-2201, JWH-018 N-(5-hydroxypentyl), and JWH-018 $N$-pentanoic acid concentrations occurred at 1.3, 2.4, and 6.5 hours, respectively. Concentrations of AM-2201, JWH-018 $\mathrm{N}$-(5-hydroxypentyl), and $\mathrm{JWH}-018 \mathrm{~N}$-pentanoic acid were negatively correlated with body temperature, but, given the low concentrations of metabolites detected, AM-2201 is likely the major contributor to pharmacodynamic effects under our experimental conditions.

\section{Introduction}

[1-(5-Fluoropentyl)-1H-indol-3-yl](1-naphthyl)methanone (AM-2201) is a synthetic cannabinoid that was first developed in 2000 as a pharmacological tool to study the endocannabinoid system (Fig. 1) (Makriyannis and Deng, 2000). AM-2201 is a full agonist at cannabinoid-1 receptors $\left(\mathrm{CB}_{1} \mathrm{Rs}\right)$ (Chimalakonda et al., 2012), producing psychoactive effects similar to the phytocannabinoid $\Delta^{9}$-tetrahydrocannabinol (Huestis et al., 2001), but with a binding affinity 40 times higher (Makriyannis and Deng, 2000; Chimalakonda et al., 2012). Similarly, the binding affinity of AM-2201 at cannabinoid-2 receptors, which

This study was supported by the Intramural Research Program of the National Institute on Drug Abuse, National Institutes of Health Grant [DA00523].

${ }^{1}$ Current affiliation: CIAN Diagnostics L.L.C., Frederick, Maryland.

${ }^{2}$ Current affiliation: Department of Forensic Genetics and Forensic Toxicology, National Board of Forensic Medicine, Linköping, Sweden.

${ }^{3}$ Current affiliation: The Lambert Center for the Study of Medicinal Cannabis and Hemp, Institute of Emerging Health Professions, Thomas Jefferson University, Philadelphia, Pennsylvania.

https://doi.org/10.1124/jpet.118.250530. are responsible for cannabinoid-mediated peripheral effects, is 14 times higher than that of $\Delta^{9}$-tetrahydrocannabinol (Makriyannis and Deng, 2000). Smoking is the predominant route of AM-2201 consumption, and typical smoked doses of $250 \mu \mathrm{g}$ to $2 \mathrm{mg}$ are estimated from online drug forums (e.g., https://drugs-forum.com/wiki/AM-2201). By contrast, a 5-mg AM-2201 oral dose was reported as a "comfortable entry dose," whereas $10 \mathrm{mg}$ would be "powerful" (https://drugsforum.com/wiki/AM-2201). These dose differences indicate potential AM-2201 degradation in the stomach, limited gastrointestinal absorption, or extensive gastrointestinal or hepatic metabolism. AM-2201 consumption induces typical cannabimimetic effects such as dry mouth, nausea, drowsiness, confusion, mydriasis, and tachycardia (Holm et al., 2013; Celofiga et al., 2014; Elian and Hackett, 2014; Musshoff et al., 2014; WHO, 2014). At higher doses, psychiatric and autonomic complications, such as extreme anxiety, acute psychosis, hyperemesis, and convulsions are reported (Patton et al., 2013; Celofiga et al., 2014).

Considering the potential risks associated with AM-2201 intake, pharmacodynamic and pharmacokinetic studies are

ABBREVIATIONS: AM-2201, 1-[(5-fluoropentyl)-1H-indol-3-yl]-(naphthalen-1-yl)methanone; AUC, area under the curve; $\mathrm{CB}_{1} \mathrm{R}$, cannabinoid-1 receptor; JWH-018, naphthalen-1-yl-(1-pentylindol-3-yl)methanone; JHW-073, naphthalen-1-yl-(1-butylindol-3-yl)methanone; LC-MS/MS, liquid chromatography-tandem mass spectrometry; NIDA, National Institute on Drug Abuse; $t_{1 / 2}$, elimination half-life; $T_{\max }$, time of maximum concentration. 


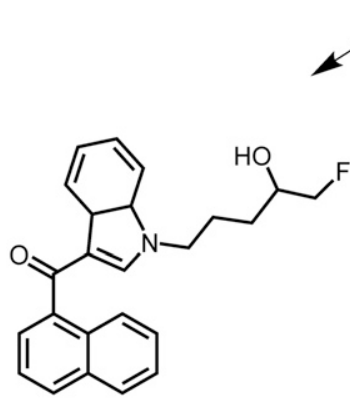

AM-2201 N-(4hydroxypentyl)
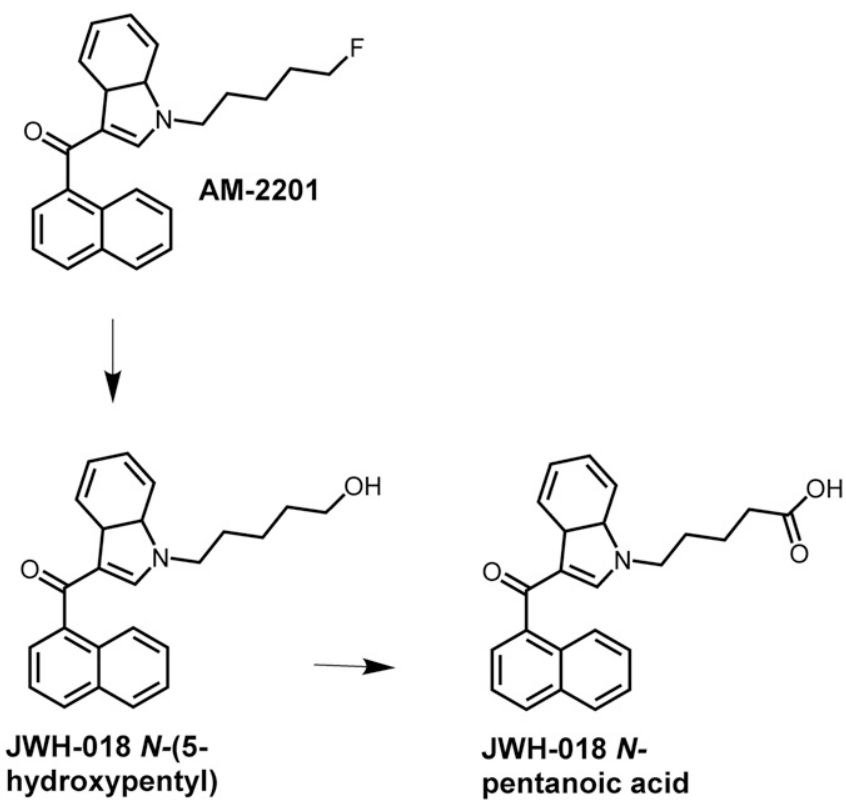

Fig. 1. Chemical structures of AM-2201 and its major metabolites. needed to document consumption in clinical and forensic cases. In vitro and in vivo animal and human experiments identified six major AM-2201 metabolites: AM-2201 N-(4hydroxypentyl), AM-2201 6'-hydroxyindole, naphthalen-1-yl(1-pentylindol-3-yl)methanone (JWH-018) $N$-(5-hydroxypentyl), JWH-018 $N$-pentanoic acid, naphthalen-1-yl-(1-butylindol-3yl)methanone (JWH-073) $N$-(4-hydroxybutyl), and JWH-073 $N$-butanoic acid (Chimalakonda et al., 2012; Sobolevsky et al., 2012; Hutter et al., 2013; Elian and Hackett, 2014; Jang et al., 2014; Kim et al., 2015). AM-2201 and metabolite blood concentrations generally range from $<0.1$ to $12 \mu \mathrm{g} / \mathrm{l}$ in humans, with JWH-018 N-(5-hydroxypentyl) and JWH-018 $N$-pentanoic acid as principal metabolites (Fig. 1) (Holm et al., 2013; Hutter et al., 2013; Kneisel et al., 2013; Patton et al., 2013; Wikstrom et al., 2013; Yeakel and Logan, 2013; Musshoff et al., 2014; Tuv et al., 2014; World Health Organization, 2014). Importantly, AM-2201 is not detected in human urine. In a preclinical study, AM-2201 6'-hydroxyindole and JWH-018 $N$-pentanoic acid were the main AM-2201 metabolites in rat urine after repeated intraperitoneal injections of high drug doses (15 mg/kg) (Jang et al., 2014). To date, only one AM-2201 pharmacokinetic study is reported in the literature. AM-2201 kinetics were reported from a single case of human oral self-administration $(0.07 \mathrm{mg} / \mathrm{kg})$, where AM-2201 and metabolite elimination from blood and urine were followed for up to 267 hours postadministration. However, AM-2201 and JWH-018 N-(5-hydroxypentyl) kinetic profiles were incomplete as maximum concentrations were not captured (Hutter et al., 2013).

Pharmacodynamic effects of cannabinoids in rodents can be evaluated by standard tests examining physiologic parameters and behavioral endpoints. The "cannabinoid tetrad" is a set of tests to identify $\mathrm{CB}_{1} \mathrm{R}$ activation that usually includes the measurement of hypothermia, catalepsy, reduction in motor activity, and antinociceptive effects (Smith et al., 1994; Ovadia et al., 1995; McGregor et al., 1996; Stein et al., 1996; Rawls et al., 2002; Brents et al., 2011, 2012; Banister et al., 2015a,b). In 2015, the first AM-2201 study in rats demonstrated that intraperitoneal injection of $0.3,1.0$, and
$3.0 \mathrm{mg} / \mathrm{kg}$ produced robust dose-related hypothermia (Banister et al., 2015b), and we confirmed these findings using subcutaneous administration of the drug to rats (Schindler et al., 2017). The metabolites AM-2201 $N$-(4-hydroxypentyl) and JWH-018 $\mathrm{N}$-(5-hydroxypentyl) are known to be $\mathrm{CB}_{1} \mathrm{R}$ full agonists (Brents et al., 2011; Chimalakonda et al., 2012), and these compounds may contribute to the effects of AM-2201 consumption. The relationships between AM-2201 metabolite blood concentrations and cannabinoid effects has yet to be determined. Given the scarcity of pharmacological data for AM-2201, we investigated AM-2201 pharmacodynamics and pharmacokinetics after injections of AM-2201 $(0.1,0.3$, and $1.0 \mathrm{mg} / \mathrm{kg}$, s.c.) in male rats fitted with temperature transponders and indwelling jugular catheters. We measured body temperature and catalepsy at timed intervals postinjection, while simultaneously obtaining serial blood specimens for analysis of AM-2201 and its metabolites using liquid chromatography tandem mass spectrometry (LC-MS/MS) (Carlier et al., 2016).

\section{Materials and Methods}

Chemical and Reagents. Analytical standards were purchased from Cayman Chemical (Ann Arbor, MI) and stored at $-20^{\circ} \mathrm{C}$ until use. AM-2201 for administration to rats was provided by the National Institute on Drug Abuse (NIDA) Drug Supply Program (Rockville, MD). LC-MS-grade water, methanol, and formic acid (Optima LC/MS) were obtained from Thermo Fisher Scientific (Waltham, MA). LC-MS-grade acetonitrile, high-performance liquid chromatography-grade tert-butyl methyl ether, dimethyl sulfoxide, sodium metabisulfite, and Tween 80 were acquired from Sigma-Aldrich (St. Louis, MO), whereas sterile $0.9 \% \mathrm{NaCl}$ (saline) was obtained from Hospira, Inc (Lake Forest, IL). Heparin saline (1000 IU/ml) was purchased from Thomas Scientific (Swedesboro, NJ), and sodium pentobarbital was furnished by the NIDA Intramural Research Program Pharmacy (Baltimore, MD). Distilled water was produced by an ELGA PURELAB Ultra Analytic Purifier (Siemens Water Technologies, Lowell, MA). BG-100 Red abalone enzyme solution from KURA Biotec (Puerto Varas, Chile) was diluted in distilled water to contain $15,625 \mathrm{U} / \mathrm{ml}$ glucuronidase and $1250 \mathrm{U} / \mathrm{ml}$ sulfatase. Ammonium acetate buffer was prepared 
with ammonium acetate salt (Sigma-Aldrich) dissolved in distilled water; $\mathrm{pH}$ was subsequently adjusted with glacial acetic acid (Thermo Fisher Scientific).

Subjects. Male Sprague-Dawley rats (weight, 300-400 g) were purchased from Harlan Laboratories (Frederick, MD). Subjects were double housed under conditions of controlled temperature $\left(22 \pm 2^{\circ} \mathrm{C}\right)$ and humidity $(45 \% \pm 5 \%)$ with ad libitum access to food and water. Lights were on between 7:00 AM and 7:00 PM. The NIDA Intramural Research Program Animal Care and Use Committee approved the animal experiments, and all procedures were carried out in accordance with the National Institutes of Health Guide for the Care and Use of Laboratory Animals. Vivarium facilities were fully accredited by the Association for Assessment and Accreditation of Laboratory Animal Care. Experiments were designed to minimize the number of animals included in the study.

Surgical Procedures. Rats were anesthetized with sodium pentobarbital (60 mg/kg, i.p.), and catheters constructed of Silastic (Dow Corning, Midland, MI) and vinyl tubing were surgically implanted into the right jugular vein as previously described (Concheiro et al., 2014). Briefly, the proximal Silastic end of the catheter was advanced to the atrium while the distal vinyl end was exteriorized on the nape of the neck and plugged with a metal stylet. Immediately after catheter implantation, while still under anesthesia, rats received surgically implanted temperature transponders (model IPTT-300; Bio Medic Data Systems, Seaford, DE) to allow for the noninvasive measurement of body temperature (Elmore and Baumann, 2018). The temperature transponder emits radio frequency signals that are received by a compatible handheld reader system (DAS-7006/7r; Bio Medic Data Systems). Transponders are cylindrical in shape, $14 \times 2 \mathrm{~mm}$, and were implanted subcutaneously along the midline of the back posterior to the shoulder blades via a prepackaged sterile guide needle delivery system. Rats were single housed postoperatively and given at least 1 week to recover from surgery.

Blood Collection Procedures and AM-2201 Injections. On the day of an experiment, rats were brought into the laboratory in their home cages and allowed 1 hour to acclimate to the surroundings. Polyethylene extension tubes were attached to 1 -ml tuberculin syringes, filled with sterile saline, and connected to the vinyl end of the catheters. The extension tubes were threaded outside the cage to facilitate blood sampling by an investigator remote from the animal. Catheters were flushed with $0.3 \mathrm{ml}$ of $48 \mathrm{IU} / \mathrm{ml}$ heparin saline to facilitate blood withdrawal. To prepare drug solutions, each milligram of AM-2201 was diluted into $50 \mu$ l of dimethylsulfoxide and $50 \mu \mathrm{l}$ of Tween 80 , then sonicated for 1 minute to dissolve. To this solution, $900 \mu \mathrm{l}$ of sterile saline was added to yield a $1 \mathrm{mg} / \mathrm{ml}$ stock solution of AM-2201. Aliquots of stock solution were diluted with vehicle consisting of dimethylsulfoxide/Tween 80/saline at 1:1: $18(\mathrm{v} / \mathrm{v} / \mathrm{v})$ to yield drug concentrations of 0.3 and $0.1 \mathrm{mg} / \mathrm{ml}$. Groups of rats received subcutaneous injection of vehicle (control), or 0.1, 0.3 , or $1.0 \mathrm{mg} / \mathrm{kg} \mathrm{AM}-2201$ on the lower back between the hips. The subcutaneous route of administration was chosen because our previous studies used this route when examining the effects of AM-2201 and other cannabinoids in rats (Schindler et al., 2017; Elmore and Baumann, 2018). Additionally, similar to the smoked route of administration, the subcutaneous route largely bypasses the first-pass metabolism in the liver. Rats were randomly assigned to each dose group. Blood specimens $(300 \mu \mathrm{l})$ were withdrawn via catheters immediately before $\left(t_{0}\right)$ and at $0.25,0.5,1,2,4,8$, and 24 hours after subcutaneous injection. Specimens were collected into 1-ml tuberculin syringes, then transferred to $1.5-\mathrm{ml}$ plastic tubes containing $5 \mu \mathrm{l}$ of $250 \mathrm{mM}$ sodium metabisulfite as a preservative and $5 \mu \mathrm{l}$ of $1000 \mathrm{IU} / \mathrm{ml}$ heparin as an anticoagulant. Blood was centrifuged at $1000 \mathrm{~g}$ for 10 minutes at $4^{\circ} \mathrm{C}$. Plasma was decanted into cryovials and stored at $-80^{\circ} \mathrm{C}$ until analysis. After each blood withdrawal, an equal volume of saline solution was infused via the intravenous catheter to maintain volume and osmotic homeostasis. Rats were free to move around the cage during the sampling procedure.
Measurement of Catalepsy and Body Temperature. Catalepsy scores and body temperature were determined at each blood withdrawal. Rat behaviors were observed by an experienced rater for 1 minute just prior to the measurement of body temperature via a handheld reader sensitive to signals emitted by the surgically implanted transponder. The behavioral rater was blind to treatment conditions. On each test day, one investigator prepared drug solutions and administered the drug to rats, whereas another investigator performed the behavioral scoring without knowing the dose administered to each subject. During the 1-minute observation period, catalepsy behaviors were scored, as previously described, based on the following three overt symptoms: immobility, flattened body posture, and splayed limbs (Elmore and Baumann, 2018). Each symptom was scored as $1=$ absent, $2=$ present, or $3=$ continuous or intense, at each time point. For each rat, catalepsy scores at each time point were summed, yielding a minimum score of 3 and a maximum score of 9 . Blood samples were withdrawn immediately after temperature recording.

AM-2201 and Metabolites Quantification. Plasma specimens were assayed using a fully validated analytical method capable of detecting and quantifying AM-2201 and 13 of its metabolites: AM-2201 N-(4-hydroxypentyl), AM-2201 6'-hydroxyindole, AM-2201 7'-hydroxyindole, JWH-018 N-(2-hydroxypentyl), JWH-018 N-(3hydroxypentyl), JWH-018 $N$-(4-hydroxypentyl), JWH-018 $N$-(5hydroxypentyl), JWH-018 $N$-pentanoic acid, JWH-018 $N$-propanoic acid, JWH-073 $N$-(2-hydroxybutyl), JWH-073 $N$-(3-hydroxybutyl), JWH-073 $N$-(4-hydroxybutyl), and JWH-073 $N$-butanoic acid (Carlier et al., 2016). Briefly, 75- $\mu$ l aliquots were hydrolyzed in $400 \mathrm{mM}$ ammonium acetate buffer, $\mathrm{pH} 4.0$, with $\beta$-glucuronidase and sulfatase enzymes. Samples were diluted in acetonitrile and ammonium acetate buffer then poured onto supported liquid extraction cartridges (1-ml ISOLUTE SLE + cartridges; Biotage, Charlotte, NC). Analytes were eluted with tert-butyl methyl ether, and solvent was evaporated under a nitrogen stream. Residues were reconstituted in mobile phase before injection onto the chromatographic system.

Analysis was performed by LC-MS/MS with a Shimadzu system consisting of an LC-30AD high-performance liquid chromatograph coupled to an LC-MS-8050 mass spectrometer (Shimadzu Corp., Columbia, MD). MS transitions were monitored as follows (quantification transition is in bold): $\mathbf{3 6 0}>\mathbf{1 2 7}$ and $360>155$ for AM-2201; $\mathbf{3 7 6}>\mathbf{1 2 7 . 1}$ and $376>155.1$ for AM-2201 $N$-(4-hydroxypentyl); $376>$ 127 and $376>155$ for AM-2201 6'-hydroxyindole; $376>127$ and $376>155$ for AM-2201 7'-hydroxyindole; 358 > 155 and $358>127$ for JWH-018 $N$-(2-hydroxypentyl); $358>155$ and $358>127$ for JWH-018 $N$-(3-hydroxypentyl); $358>\mathbf{1 5 5}$ and $358>127$ for JWH-018 $N$-(4hydroxypentyl); $358>155$ and $358>127$ for JWH-018 $N$-(5hydroxypentyl); $372>155$ and $372>127$ for JWH-018 $N$-pentanoic acid; $344>155$ and $344>127$ for JWH-018 $N$-propanoic acid; $344>$ 155 and $344>127$ for JWH-073 $N$-(2-hydroxybutyl); $344>127$ and $344>155$ for JWH-073 $N$-(3-hydroxybutyl); $344>127$ and $344>$ 155 for JWH-073 $N$-(4-hydroxybutyl); and 358 > 155 and $358>127$ for JWH-073 $N$-butanoic acid. Data were processed with ASCENT software from Indigo BioAutomation (Indianapolis, IN). Gradient elution was performed at $700 \mu \mathrm{l} / \mathrm{min}$ on a Raptor LC biphenyl column (Restek, Bellefonte, PA) with mobile phase A $(0.1 \%$ formic acid in water) and mobile phase B [0.1\% formic acid in methanol/acetonitrile $50: 50(\mathrm{v} / \mathrm{v})]$. Two multiple reaction monitoring mass transitions were monitored for each analyte and internal standard.

Each sample batch was accompanied by seven calibrators, a blank; a blank fortified with internal standards; and low-, medium-, and high-quality controls (Carlier et al., 2016). The lowest limits of quantification were $0.1 \mu \mathrm{g} / \mathrm{l}$ for AM-2201 $N$-(4-hydroxypentyl), JWH$018 N$-propanoic acid, and JWH-073 $N$-(2-hydroxybutyl), and $0.05 \mu \mathrm{g} / 1$ for other analytes. Analyte recoveries and matrix effects were $58.4 \%$ $84.4 \%$ and $-62.1 \%$ to $-15.6 \%$, respectively $(n=10)$. Interassay bias and imprecision were $88.8 \%-110.1 \%$ and $0.3 \%-11.9 \% \mathrm{CVs}$, respectively $(n=10)$. 
Data Analysis and Statistics. Pharmacodynamic and pharmacokinetic findings were statistically evaluated with GraphPad Prism version 7.04 (GraphPad Software, La Jolla, CA). Body temperature and catalepsy data were evaluated with two-way analysis of variance (dose $\times$ time) followed by Bonferroni post hoc tests. Pharmacokinetic data were analyzed using WinNonlin version 5.2 (Pharsight, Mountain View, CA) to calculate pharmacokinetic constants, including $C_{\max }$, time of maximum concentration $\left(\mathrm{T}_{\max }\right)$, area under the curve (AUC), and elimination half-life $\left(t_{1 / 2}\right)$ for each analyte. We examined the AUC from 0 to 24 hours postinjection for AM-2201 and its metabolites. AUC is the integral of the time-concentration profile for a given analyte and represents the total analyte exposure over time. The pharmacokinetic constants were subjected to one-way analysis of variance (dose) followed by Bonferroni post hoc tests to determine differences between dose groups. At least three data points on the terminal elimination phase were required for $t_{1 / 2}$ determination. Relationships between analyte plasma concentrations and body temperature were assessed using a Pearson's correlation analysis. $P<0.05$ was used as the minimum statistical significance threshold for all comparisons.

\section{Results}

Pharmacodynamic Effects. The time-course effects for body temperature and catalepsy after subcutaneous AM-2201 administration $(0.1,0.3$, and $1.0 \mathrm{mg} / \mathrm{kg})$ are depicted in Fig. 2. Temperature was significantly affected by AM-2201 dose $\left(F_{3,16}=13.96, P<0.0001\right)$ and time $\left(F_{7,112}=65.00, P<\right.$ $0.0001)$, with a significant dose $\times$ time interaction $\left(F_{21,112}=\right.$ 14.18, $P<0.0001$ ). Specifically, AM-2201 produced a doserelated reduction in body temperature compared with vehicle control that lasted up to 8 hours postinjection. The maximum temperature drop occurred at 2 hours postinjection for all doses, with $1.4,2.0$, and $4.0^{\circ} \mathrm{C}$ decreases at $0.1,0.3$, and $1.0 \mathrm{mg} /$ $\mathrm{kg}$, respectively. After the $0.1 \mathrm{mg} / \mathrm{kg}$ dose of AM-2201, temperature decreased significantly compared with control only at 2 hours postinjection, whereas after the $1.0 \mathrm{mg} / \mathrm{kg}$ dose, temperature decreased for 8 hours but returned to baseline by 24 hours. Catalepsy scores were significantly affected by AM-2201 dose $\left(F_{3,16}=119.30, P<0.0001\right)$ and time after drug administration $\left(F_{7,112}=32.47, P<0.0001\right)$, with a significant dose $\times$ time interaction $\left(F_{21,112}=8.36, P<0.0001\right)$. AM-2201 induced a dose-related increase in catalepsy scores that lasted up to 4 hours postinjection. The maximum increase in catalepsy occurred at 1 hour postinjection for all doses. After a dose of $0.1 \mathrm{mg} / \mathrm{kg}$ AM-2201, catalepsy increased above control levels only at the 1 -hour time point, whereas after the
$1.0 \mathrm{mg} / \mathrm{kg}$ dose, catalepsy increased at all time points for 4 hours postinjection.

AM-2201 and Metabolite Pharmacokinetics. The timeconcentration profiles for AM-2201 and its metabolites are depicted in Fig. 3. It is noteworthy that the assay method we used for this study was designed to detect and quantify AM-2201 and 13 of its identified metabolites (Carlier et al., 2016). Nevertheless, only three metabolites, JWH-018 N-(5hydroxypentyl), JWH-018 $N$-pentanoic acid, and AM-2201 $N$ (4-hydroxypentyl), were detected in rat plasma specimens from our studies. Furthermore, AM-2201 $N$-(4-hydroxypentyl) was detected in only five samples at 2,4 , and 8 hours after the administration of 0.3 and $1.0 \mathrm{mg} / \mathrm{kg}$ AM-2201, respectively. Given the small number of AM-2201 $N$-(4-hydroxypentyl)positive specimens, this metabolite was excluded from pharmacokinetic statistical analysis. Pharmacokinetic constants are reported in Table 1. Plasma time-concentration profiles for AM-2201 were significantly affected by dose $\left(F_{2,11}=15.67\right.$, $P<0.001)$ and time $\left(F_{6,66}=12.69, P<0.0001\right)$, with concentrations rising linearly as dose increased. AM-2201 concentrations after a dose of $0.3 \mathrm{mg} / \mathrm{kg}$ were significantly greater than those after $0.1 \mathrm{mg} / \mathrm{kg}$ at 0.5 and 1 hour postinjection, whereas concentrations after $1.0 \mathrm{mg} / \mathrm{kg}$ were greater than those after $0.1 \mathrm{mg} / \mathrm{kg}$ for 8 hours postinjection. AM-2201 was still detectable 24 hours after the injection of $1.0 \mathrm{mg} / \mathrm{kg}$, with a value of $3.22 \pm 0.40 \mu \mathrm{g} / \mathrm{l}$. AM-2201 $C_{\max }$ was significantly altered by the dose administered $\left(F_{2,11}=6.39, P<\right.$ $0.01)$, as was AUC $\left(F_{2,11}=34.84, P<0.001\right)$. Post hoc tests revealed that AM-2201 $C_{\max }$ and AUC values after administration of a $1.0 \mathrm{mg} / \mathrm{kg}$ dose were significantly greater than those observed for the 0.3 and $0.1 \mathrm{mg} / \mathrm{kg}$ doses. AM-2201 $\mathrm{T}_{\max }$ was achieved at $\sim 1.3$ hours and not affected by the dose administered $\left(F_{2,11}=0.03\right.$, nanosecond $)$. By contrast, AM-2201 $t_{1 / 2}$ was influenced by dose $\left(F_{2,11}=5.54, P<0.02\right)$, such that $t_{1 / 2}$ after $1.0 \mathrm{mg} / \mathrm{kg}$ was significantly greater than that observed after the $0.1 \mathrm{mg} / \mathrm{kg}$ dose.

JWH-018 $N$-(5-hydroxypentyl) plasma time-concentration profiles were significantly affected by dose $\left(F_{2,11}=67.29, P<\right.$ $0.0001)$ and time $\left(F_{6,66}=48.53, P<0.0001\right)$, with concentrations rising as dose increased (Fig. 3). JWH-018 $N$-(5-hydroxypentyl) concentrations after a dose of $0.3 \mathrm{mg} / \mathrm{kg}$ AM-2201 were significantly greater than those after $0.1 \mathrm{mg} / \mathrm{kg}$ at 1,2 , and 4 hours postinjection, whereas concentrations after a $1.0 \mathrm{mg} / \mathrm{kg}$ dose were greater than those after $0.1 \mathrm{mg} / \mathrm{kg}$ for all time points up to 8 hours postinjection. JWH-018 N-(5-
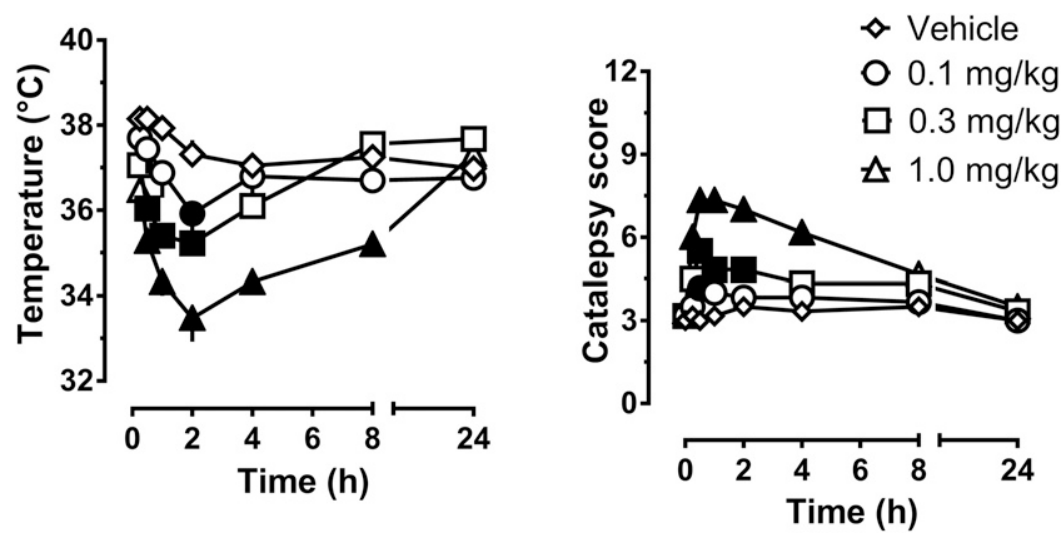

Fig. 2. Dose-response effects of subcutaneously administered AM-2201 on body temperatures and catalepsy scores in male rats. Rats fitted with indwelling jugular catheters and temperature transponders received vehicle $(1.0 \mathrm{ml} / \mathrm{kg}$, s.c.) or AM-2201 $(0.1,0.3$, or $1.0 \mathrm{mg} / \mathrm{kg}$, s.c. $)$ at time zero. Temperatures were recorded, and behaviors were scored just prior to blood sampling at $0.25,0.5,1,2,4,8$, and 24 hours after injection. Values are expressed as the mean \pm S.E.M. for $N=4-5$ rats/group. Filled symbols indicate significant differences compared with vehicle at a given time point, $P<0.05$. 

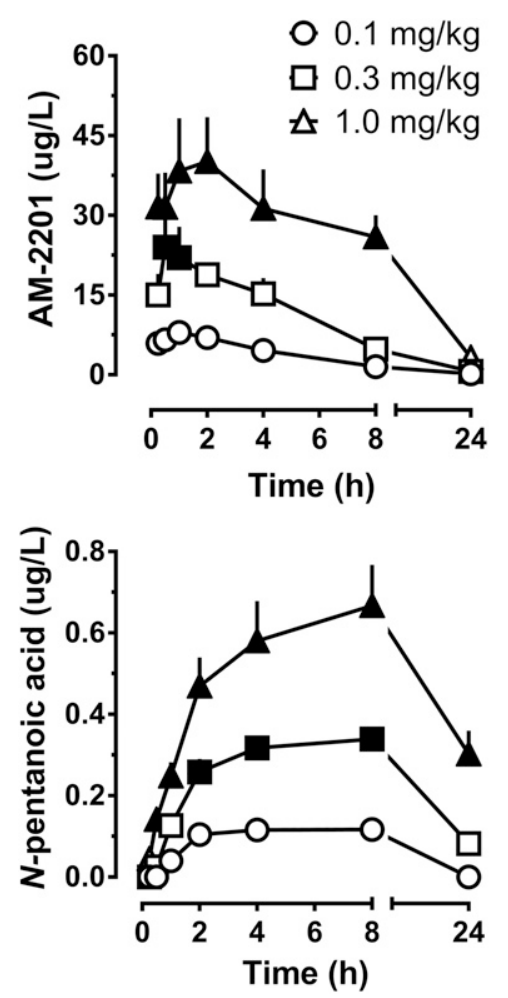
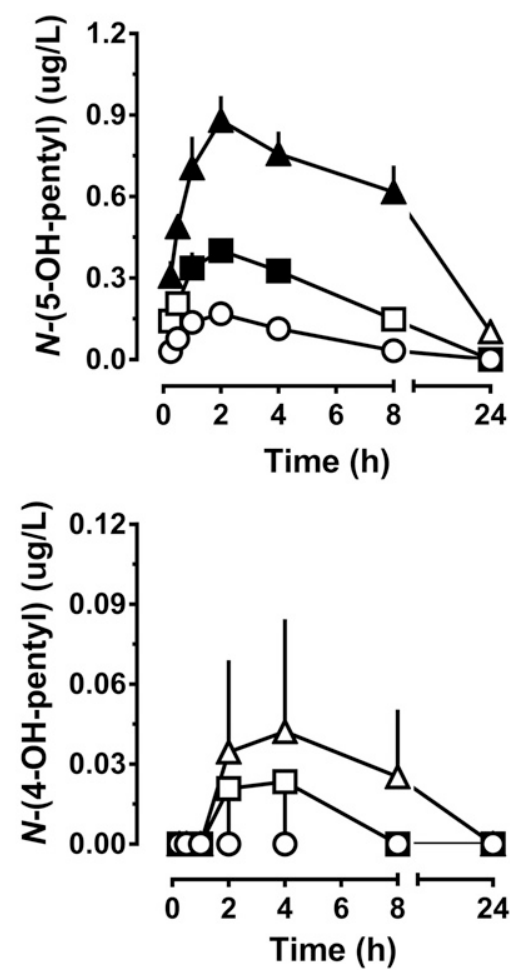

Fig. 3. Time-concentration profiles for AM-2201, JWH-018 $N$-(5-hydroxypentyl), JWH-018 $N$-pentanoic acid, and AM-2201 N-(4-hydroxypentyl) in rats receiving AM-2201 administered subcutaneously. Rats fitted with indwelling jugular catheters and temperature transponders received AM-2201 doses of $0.1,0.3$, or $1.0 \mathrm{mg} / \mathrm{kg}$ s.c. at time zero. Blood samples were withdrawn via the catheters at 0.25 , $0.5,1,2,4,8$, and 24 hours after injection, and plasma specimens were assayed for analytes using LC-MS/MS. Data are for mean \pm S.E.M. for $N=4-5$ rats/group. Filled symbols indicate significant differences compared with the low dose administered $(0.1 \mathrm{mg} / \mathrm{kg})$ at a given time point, $P<$ 0.05 . hydroxypentyl) was measurable 24 hours after $1.0 \mathrm{mg} / \mathrm{kg}$ AM-2201, but concentrations were close to the limits of detection. JWH-018 $N$-(5-hydroxypentyl) $C_{\max }$ was significantly augmented as dose increased $\left(F_{2,11}=51.57, P<\right.$ $0.001)$ and AUC was influenced in a similar manner $\left(F_{2,11}=\right.$ 104.90, $P<0.001)$. Post hoc tests revealed that $N$-(5-hydroxypentyl) $C_{\max }$ and AUC values after a dose of $1.0 \mathrm{mg} / \mathrm{kg}$ were significantly above those observed for 0.1 and $0.3 \mathrm{mg} / \mathrm{kg}$. JWH$018 N$-(5-hydroxypentyl) $\mathrm{T}_{\max }$ occurred at $\sim 2$ hours and was not affected by the dose administered $\left(F_{2,11}=1.63\right.$, nanosecond), whereas $t_{1 / 2}$ for this metabolite could not be determined due to the lack of data from the descending limb of the elimination curve. JWH-018 N-pentanoic acid concentration-time profiles were significantly affected by $\operatorname{dose}\left(F_{2,11}=39.42, P<0.0001\right)$ and time $\left(F_{6,66}=73.86, P<\right.$ 0.0001 , with concentrations rising as dose increased. JWH-018
$N$-pentanoic acid concentrations after a dose of $0.3 \mathrm{mg} / \mathrm{kg}$ AM-2201 were significantly greater than those after $0.1 \mathrm{mg} / \mathrm{kg}$ at 2,4 , and 8 hours postinjection, whereas concentrations after $1.0 \mathrm{mg} / \mathrm{kg}$ were greater than those after $0.1 \mathrm{mg} / \mathrm{kg}$ for all time points up to 8 hours postinjection. JWH-018 $N$-pentanoic acid was detectable 24 hours after $1.0 \mathrm{mg} / \mathrm{kg}$ AM-2201, with a concentration of $0.31 \pm 0.06 \mu \mathrm{g} / \mathrm{l}$. JWH-018 $N$-pentanoic acid $C_{\max }$ was significantly greater as dose increased $\left(F_{2,11}=\right.$ $27.54, P<0.001)$ as was $\operatorname{AUC}\left(F_{2,11}=147.02, P<0.001\right)$. JWH-018 $N$-pentanoic acid $\mathrm{T}_{\max }$ was delayed compared with JWH-018 $N$-(5-hydroxypentyl) and was not affected by dose $\left(F_{2,11}=1.99\right.$, nanosecond $)$, and $t_{1 / 2}$ for this metabolite could not be determined.

Correlation Analyses. Because we measured pharmacodynamic and pharmacokinetic endpoints from the same rats, we were able to examine relationships between temperature

TABLE 1

Pharmacokinetic constants for AM-2201 and its metabolites after subcutaneous administration of 0.1, 0.3 , or $1.0 \mathrm{mg} / \mathrm{kg}$ AM-2201 in male rats

Data are expressed as the mean \pm S.E.M. for $N=4-5$ rats/group.

\begin{tabular}{|c|c|c|c|c|c|}
\hline \multirow{2}{*}{ Analyte } & AM-2201 Dose & $C_{\max }$ & AUC & $\mathrm{T}_{\max }$ & $t_{1 / 2}$ \\
\hline & $\mathrm{mg} / \mathrm{kg}$ & $\mu \mathrm{g} / \mathrm{l}$ & $\mathrm{h} / \mu \mathrm{g}$ per liter & \multicolumn{2}{|c|}{$\mathrm{h}$} \\
\hline \multirow[t]{3}{*}{ AM-2201 } & $0.1(N=5)$ & $8.2 \pm 1.7$ & $51 \pm 8$ & $1.4 \pm 0.2$ & $4.3 \pm 0.1$ \\
\hline & $0.3(N=5)$ & $33 \pm 8.9$ & $149 \pm 13$ & $1.3 \pm 0.3$ & $4.6 \pm 0.3$ \\
\hline & $1.0(N=4)$ & $42 \pm 9^{*}$ & $487 \pm 74^{* *}$ & $1.3 \pm 0.4$ & $6.3 \pm 0.8^{*}$ \\
\hline \multirow{3}{*}{ JWH-018 N-(5-hydroxypentyl) } & $0.1(N=5)$ & $0.17 \pm 0.02$ & $0.71 \pm 0.11$ & $1.8 \pm 0.2$ & N.D. \\
\hline & $0.3(N=5)$ & $0.40 \pm 0.04$ & $2.1 \pm 0.1$ & $2.0 \pm 0.0$ & N.D. \\
\hline & $1.0(N=4)$ & $0.91 \pm 0.08^{* *}$ & $11 \pm 1^{* *}$ & $3.5 \pm 1.5$ & N.D. \\
\hline \multirow[t]{3}{*}{ JWH-018 N-pentanoic acid } & $0.1(N=5)$ & $0.12 \pm 0.01$ & $0.77 \pm 0.09$ & $4.8 \pm 1.3$ & N.D. \\
\hline & $0.3(N=5)$ & $0.36 \pm 0.02$ & $5.4 \pm 0.4^{*}$ & $6.8 \pm 1.2$ & N.D. \\
\hline & $1.0(N=4)$ & $0.67 \pm 0.10 * *$ & $12.0 \pm 1.5^{* *}$ & $8.0 \pm 0.8$ & N.D. \\
\hline
\end{tabular}

N.D., not determined.

${ }^{*} P<0.05$, significant difference compared with $0.1 \mathrm{mg} / \mathrm{kg}$ dose; $* * P<0.05$, significant difference compared with 0.1 and $0.3 \mathrm{mg} / \mathrm{kg}$ doses. 
and analyte concentrations in plasma. The correlation findings are depicted in Fig. 4. Body temperature was negatively correlated to AM-2201 (Pearson's $r=-0.608, P<0.0001$ ), JWH-018 N-(5-hydroxypentyl) (Pearson's $r=-0.778, P<$ 0.0001 ), and JWH-018 $N$-pentanoic acid (Pearson's $r=-0.379$, $P<0.001$ ) plasma concentrations.

\section{Discussion}

AM-2201 is an example of a synthetic compound that was initially developed as a pharmacological tool to investigate the endocannabinoid system but was subsequently diverted for recreational use in humans. The compound was one of the most common synthetic cannabinoids found in plant-based "spice" products available in the clandestine market during 2011-2013 in the United States (https://www.deadiversion. usdoj.gov/nflis/spec_rpt_CathCan_2013.pdf). Here we report the pharmacodynamic effects of AM-2201 in rats, along with the first plasma pharmacokinetic profiles for the parent compound and its metabolites in vivo. Our study has three main findings. First, AM-2201 produced robust dosedependent hypothermia and catalepsy in rats, consistent with the effects of the compound reported in the literature (Banister et al., 2015b; Schindler et al., 2017). Second, only three of the previously identified metabolites of AM-2201 were found in plasma after subcutaneous administration of the drug to rats, where JWH-018 $N$-(5-hydroxypentyl) and JWH-018 $N$-pentanoic acid were the major compounds detected. Finally, the plasma concentrations of AM-2201 metabolites were far below those of the parent compound, indicating that metabolites are unlikely to contribute to the pharmacodynamic effects of the drug under the conditions of the present study.

Consistent with our temperature data, Banister et al. (2015b) demonstrated that intraperitoneal injection of AM-2201 causes transient hypothermia in rats fitted with indwelling biotelemetry transmitters. In their study, intraperitoneal administration of $0.3,1.0$, and $3.0 \mathrm{mg} / \mathrm{kg}$ AM-2201 decreased temperature $1-1.5^{\circ} \mathrm{C}$, with a maximum effect at 1 hour postinjection. A second less pronounced temperature drop occurred between 4 and 5 hours after administration $\left(\leq 0.5^{\circ} \mathrm{C}\right)$. The authors hypothesized that the second phase of AM-2201 hypothermia might be due to combined effects of the parent compound and its bioactive metabolites (Banister et al., 2015b). In our study, subcutaneous administration of AM-2201 to rats produced a dose-dependent drop in body temperature that reached a maximum at 2 hours postinjection, without any indication of a secondary delayed decrease. The subcutaneous dose of $1.0 \mathrm{mg} / \mathrm{kg}$ AM-2201 induced a hypothermic response that reached $4^{\circ} \mathrm{C}$, which is more robust than the effects observed after an equivalent intraperitoneal dose in rats (Banister et al., 2015b). The present findings agree with our previous results in rats where subcutaneous injections of AM-2201 (Schindler et al., 2017) or JWH-018 (Elmore and Baumann, 2018) induce hypothermia that is maximal after 1-2 hours and slowly returns to normal in a monophasic manner.

The absence of two-stage hypothermia in our study might be related to the subcutaneous route of administration, which would be expected to minimize AM-2201 metabolism when compared with the intraperitoneal route. We found that plasma concentrations of AM-2201, JWH-018 N-(5-hydroxypentyl), and JWH-018 $N$-pentanoic acid exhibited significant
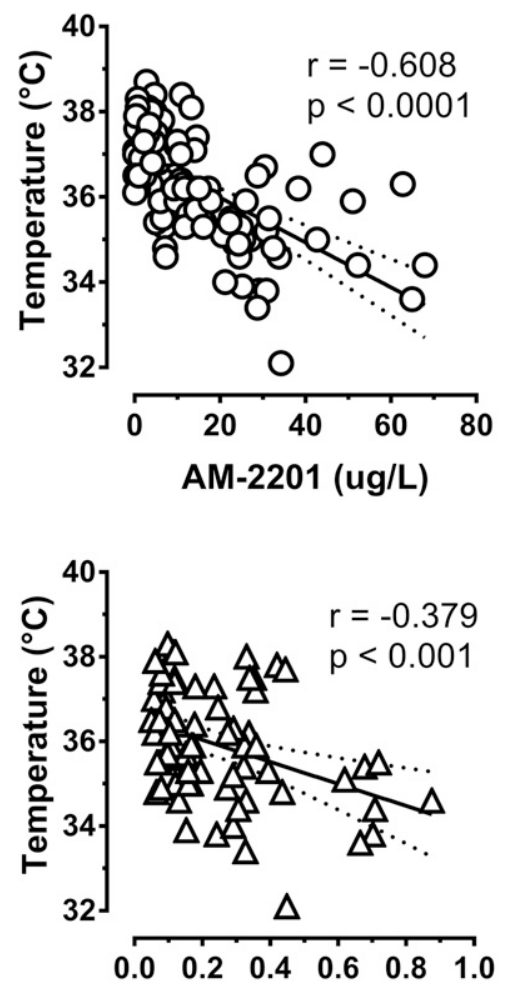

$\mathrm{N}$-pentanoic acid (ug/L)

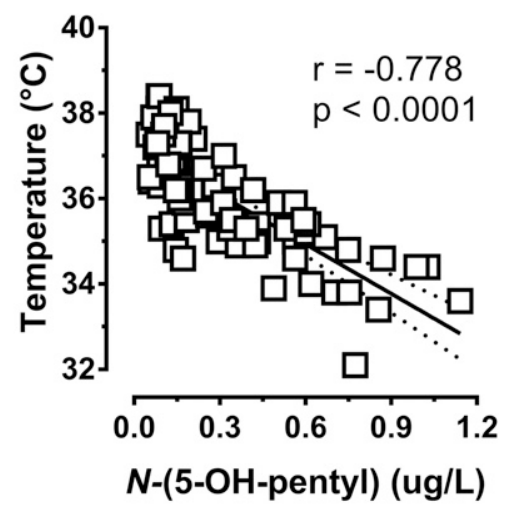

Fig. 4. Correlations between plasma concentrations of AM-2201, JWH-018 N-(5-hydroxypentyl), and JWH-018 $\mathrm{N}$-pentanoic acid vs. body temperatures. Raw data from Figs. 2 and 3 were used to construct the correlation matrices, such that analyte concentrations are plotted against temperature measures taken at the same time points. Pearson's $r$ and $P$ values are shown. 
negative correlations with body temperature, suggesting a role for these compounds in the observed hypothermic effects. AM-2201 and JWH-018 N-(5-hydroxypentyl) are both potent and efficacious $\mathrm{CB}_{1} \mathrm{R}$ agonists (Makriyannis and Deng, 2000; Brents et al., 2011; Chimalakonda et al., 2012), whereas JWH$018 \mathrm{~N}$-pentanoic acid is inactive (Brents et al., 2011). However, the low concentrations of JWH-018 N-(5-hydroxypentyl) found in the present study demonstrate this metabolite is unlikely to contribute to pharmacodynamic effects of subcutaneously administered AM-2201. It is noteworthy that human users normally self-administer AM-2201 by smoking or vaping, but we were unable to use either of these routes. The subcutaneous route was chosen because our previous studies used this route to characterize the pharmacological effects of AM-2201 and other cannabinoids in rats (Schindler et al., 2017; Elmore and Baumann, 2018). Similar to the smoked and vaped routes of administration, the subcutaneous route largely bypasses first-pass metabolism in the liver. Nevertheless, our use of the subcutaneous route limits the clinical relevance of our findings. Future studies in rodents should examine pharmacodynamic effects and pharmacokinetics of AM-2201, and other synthetic cannabinoids, after smoked or vaporized routes of administration (Lefever et al., 2017).

Our behavioral findings show for the first time that AM-2201 induces immobility, flattened body posture, and splayed limbs, which is consistent with a profound cataleptic effect lasting for 4 hours postinjection. These findings are similar to the robust catalepsy observed after the administration of JWH-018 to rats (Elmore and Baumann, 2018). In a similar manner, JWH-018 and JWH-073 induce catalepsy and locomotor suppression in mice, with maximum effects at 1 hour postadministration (3 and $10 \mathrm{mg} / \mathrm{kg}$, i.p.) (Brents et al., 2011, 2012). Taken together, the available findings agree that synthetic cannabinoids like AM-2201 and JWH-018 induce catalepsy in both rats and mice.

We present the first plasma pharmacokinetic profiles for AM-2201 and its metabolites in rats. Importantly, only 3 of the 13 metabolites of AM-2201 previously identified in vitro were detected: AM-2201 $N$-(4-hydroxypentyl), JWH-018 N-(5hydroxypentyl), and JWH-018 $N$-pentanoic acid. The analytical method we used can detect and quantify 10 other metabolites with limits of detection ranging from 0.025 to $0.100 \mu \mathrm{g} / \mathrm{l}$, but none were detected. Our findings are consistent with most in vivo experiments that identified AM-2201 N-(4hydroxypentyl), JWH-018 N-(5-hydroxypentyl), and JWH-018 $N$-pentanoic acid as major metabolites of AM-2201 in serum, urine, and hair samples of rats and humans (Chimalakonda et al., 2012; Sobolevsky et al., 2012; Hutter et al., 2013; Elian and Hackett, 2014; Jang et al., 2014; Kim et al., 2015). AM-2201 plasma concentrations and AUC values increased in proportion to the dose of AM-2201 administered, indicating linear kinetics, at least for the doses tested here. Linearity and $t_{1 / 2}$ values could not be determined for the AM-2201 metabolites because too few data points on the terminal elimination curve were included for accurate measurement. We found that AM-2201 $t_{1 / 2}$ after $1.0 \mathrm{mg} / \mathrm{kg}$ was significantly longer than $t_{1 / 2}$ after $0.1 \mathrm{mg} / \mathrm{kg}$, suggesting reduced clearance of the drug at high doses. Further studies are warranted to determine the precise $t_{1 / 2}$ for AM-2201 metabolites in rats and other species.

In 2013, Hutter et al. (2013) reported the first AM-2201 pharmacokinetic investigation in humans, based on a single case of oral consumption of the drug ( $5 \mathrm{mg}, 0.07 \mathrm{mg} / \mathrm{kg})$. In their study, AM-2201 serum concentrations decreased from 0.6 to $<0.02 \mu \mathrm{g} / \mathrm{l}$ (limit of quantification) during the 1.5-125 hours postingestion. Only 4 AM-2201 metabolites were detected in serum, including JWH-018 $N$-pentanoic acid, JWH$018 N$-(5-hydroxypentyl), AM-2201 6'-hydroxyindole, and AM-2201 $N$-(4-hydroxypentyl). Importantly, serum concentrations of JWH-018 $N$-pentanoic acid exceeded those of AM-2201 concentrations in all samples. JWH-018 $N$-(5-hydroxypentyl) and JWH-018 $N$-pentanoic acid maximum concentrations occurred at 1.5 and 4.1 hours postadministration, respectively. JWH-018 $N$-pentanoic acid was detectable up to 57 hours postingestion ( $\geq 0.05 \mu \mathrm{g} / \mathrm{l}$ ). In our rat study, AM-2201 was the major analyte in all plasma specimens after subcutaneous AM-2201 injection, whereas JWH-018 N-(5hydroxypentyl) and JWH-018 $N$-pentanoic acid were found at $>10$-fold lower concentrations. AM-2201, JWH-018 N-(5hydroxypentyl), and JWH-018 $N$-pentanoic acid $\mathrm{T}_{\max }$ values were $1.3,2.4$, and 6.5 hours, respectively, confirming early AM-2201 hydroxylation followed by later carboxylation. The high AM-2201 concentrations we measured in rats, when compared with those in the study by Hutter et al. (2013), are likely the result of interspecies or route of administration differences. In particular, Hutter et al. (2013) used oral administration of AM-2201 where gastrointestinal and hepatic metabolism would be substantial, whereas we used the subcutaneous route, which largely bypasses such metabolism.

AM-2201 blood concentrations in authentic human cases of AM-2201 exposure generally did not exceed $5 \mu \mathrm{g} / \mathrm{l}$ (Holm et al., 2013; Hutter et al., 2013; Kneisel et al., 2013; Wikstrom et al., 2013; Yeakel and Logan, 2013; Musshoff et al., 2014; Tuv et al., 2014; World Health Organization, 2014). In 2013, a man died from a self-inflicted stab wound to the neck after psychiatric complications related to AM-2201 intake; the postmortem AM-2201 blood concentration from this victim was $12 \mu \mathrm{g} / \mathrm{l}$ (Patton et al., 2013). In our rat study, the AM-2201 plasma $C_{\max }$ was $8 \mu \mathrm{g} / \mathrm{l}$ after a $0.1 \mathrm{mg} / \mathrm{kg}$ dose, which is in the range of blood concentrations reported in human users. AM-2201 plasma $C_{\text {max }}$ reached $42 \mu \mathrm{g} / \mathrm{l}$ after a $1.0 \mathrm{mg} / \mathrm{kg}$ dose, with an average concentration of $>20 \mu \mathrm{g} / \mathrm{l}$ during the 8 hours after AM-2201 injection; all animals fully recovered. In 2014, Jang et al. (2014) reported that three daily intraperitoneal injections of $15 \mathrm{mg} / \mathrm{kg}$ AM-2201 to rats led to formation of the metabolites AM-2201 $N$-(4-hydroxypentyl), AM-2201 6'hydroxyindole, JWH-018 $N$-(5-hydroxypentyl), JWH-018 $N$-pentanoic acid, and JWH-073 $N$-butanoic acid in urine. We failed to detect AM-2201 6'-hydroxyindole or JWH-073 $N$-butanoic acid in rat plasma, which could indicate rapid elimination of these metabolites into urine after formation. On the other hand, Jang et al. (2014) administered repeated injections of a much higher dose of AM-2201 than we administered here. Since we observed sustained hypothermia and catalepsy after $1.0 \mathrm{mg} / \mathrm{kg}$ AM-2201 (Fig. 2), along with high circulating concentrations of the drug, it seems that a $15 \mathrm{mg} / \mathrm{kg}$ dose of AM-2201 in rats is not relevant to doses ingested by humans.

In summary, we report the first pharmacokinetic profiles for AM-2201 and its metabolites after subcutaneous administration of the drug to rats. JWH-018 $N$-(5-hydroxypentyl) and JWH-018 $N$-pentanoic acid were the main metabolites detected, in accordance with previous preclinical, clinical, and forensic studies. In contrast to the single human case of AM-2201 ingestion, we found that AM-2201 plasma 
concentrations in rats were much higher than those of its metabolites in all samples, possibly due to interspecies differences or route of administration differences. Importantly, the AM-2201 plasma concentrations and AUC values rose linearly with increasing dose, indicating simple linear kinetics, at least for the doses tested here. Plasma concentrations of AM-2201 and its metabolites were significantly correlated with hypothermia, but the extraordinarily low concentrations of JWH$018 \mathrm{~N}$-(5-hydroxypentyl) and JWH-018 $N$-pentanoic acid indicate that these compounds do not contribute to cannabimimetic effects under our experimental conditions. Because of the paucity of human data with synthetic cannabinoid compounds, future animal studies should compare the effects of dose and route of administration on the pharmacodynamics and pharmacokinetics of these compounds as they emerge in the recreational drug marketplace.

\section{Acknowledgments}

We thank Andrew Peabody and Matt Lambing of Indigo BioAutomation for providing access to ASCENT software and assistance processing plasma data; and Shimadzu Corporation for providing LC-MS/MS instrumentation via a National Institutes of Health Materials Transfer Agreement.

\section{Authorship Contributions}

Participated in research design: Wohlfarth, Scheidweiler, Huestis, and Baumann.

Conducted experiments: Carlier, Wohlfarth, Salmeron, and Baumann.

Performed data analysis: Carlier, Scheidweiler, and Baumann.

Wrote or contributed to the writing of the manuscript: Carlier, Wohlfarth, Salmeron, Scheidweiler, Huestis, and Baumann.

\section{References}

Banister SD, Moir M, Stuart J, Kevin RC, Wood KE, Longworth M, Wilkinson SM, Beinat C, Buchanan AS, Glass M, et al. (2015a) Pharmacology of indole and indazole synthetic cannabinoid designer drugs AB-FUBINACA, ADB-FUBINACA AB-PINACA, ADB-PINACA, 5F-AB-PINACA, 5F-ADB-PINACA, ADBICA, and 5F-ADBICA. ACS Chem Neurosci 6:1546-1559.

Banister SD, Stuart J, Kevin RC, Edington A, Longworth M, Wilkinson SM, Beinat C, Buchanan AS, Hibbs DE, Glass M, et al. (2015b) Effects of bioisosteric fluorine in synthetic cannabinoid designer drugs JWH-018, AM-2201, UR-144, XLR-11, PB-22, 5F-PB-22, APICA, and STS-135. ACS Chem Neurosci 6:1445-1458.

Brents LK, Gallus-Zawada A, Radominska-Pandya A, Vasiljevik T, Prisinzano TE, Fantegrossi WE, Moran JH, and Prather PL (2012) Monohydroxylated metabolites of the K2 synthetic cannabinoid JWH-073 retain intermediate to high cannabinoid 1 receptor (CB1R) affinity and exhibit neutral antagonist to partial agonist activity. Biochem Pharmacol 83:952-961.

Brents LK, Reichard EE, Zimmerman SM, Moran JH, Fantegrossi WE, and Prather PL (2011) Phase I hydroxylated metabolites of the K2 synthetic cannabinoid JWH018 retain in vitro and in vivo cannabinoid 1 receptor affinity and activity. PLoS One 6: 21917.

Carlier J, Scheidweiler KB, Wohlfarth A, Salmeron BD, Baumann MH, and Huestis MA (2016) Quantification of [1-(5-fluoropentyl)-1H-indol-3-yl] (naphthalene-1-yl)methanone (AM-2201) and 13 metabolites in human and rat plasma by liquid chromatography-tandem mass spectrometry. $J$ Chromatogr $A$ 1451:97-106.

Celofiga A, Koprivsek J, and Klavz J (2014) Use of synthetic cannabinoids in patients with psychotic disorders: case series. J Dual Diagn 10:168-173.

Chimalakonda KC, Seely KA, Bratton SM, Brents LK, Moran CL, Endres GW, James LP, Hollenberg PF, Prather PL, Radominska-Pandya A, et al. (2012) Cytochrome P450-mediated oxidative metabolism of abused synthetic cannabinoids found in K2/Spice: identification of novel cannabinoid receptor ligands. Drug Metab Dispos 40:2174-2184
Concheiro M, Baumann MH, Scheidweiler KB, Rothman RB, Marrone GF and Huestis MA (2014) Nonlinear pharmacokinetics of (+/-)3,4-methylenedioxymethamphetamine (MDMA) and its pharmacodynamic consequences in the rat. Drug Metab Dispos 42:119-125.

Elian AA and Hackett J (2014) Analysis of AM-2201 and metabolites in a drugs and driving case. Drug Test Anal 6:389-395.

Elmore JS and Baumann MH (2018) Repeated exposure to the "spice" cannabinoid JWH-018 induces tolerance and enhances responsiveness to 5-HT1A receptor stimulation in male rats. Front Psychiatry 9:55.

Holm NB, Pineda RS, Andersen DW, Rasmussen BS, Dalsgaard PW, Hoegberg LCG Johansen SS, and Linnet K (2013) Screening of Danish traffic cases for synthetic cannabinoids in whole blood by LC-MS/MS. Scand $J$ Forensic Sci 19:45-51.

Huestis MA, Gorelick DA, Heishman SJ, Preston KL, Nelson RA, Moolchan ET, and Frank RA (2001) Blockade of effects of smoked marijuana by the CB1-selective cannabinoid receptor antagonist SR141716. Arch Gen Psychiatry 58:322-328.

Hutter M, Moosmann B, Kneisel S, and Auwärter V (2013) Characteristics of the designer drug and synthetic cannabinoid receptor agonist AM-2201 regarding its chemistry and metabolism. J Mass Spectrom 48:885-894.

Jang M, Yang W, Shin I, Choi H, Chang H, and Kim E (2014) Determination of AM-2201 metabolites in urine and comparison with JWH-018 abuse. Int $J$ Legal Med 128:285-294.

Kim J, Park Y, Park M, Kim E, Yang W, Baeck S, Lee S, and Han S (2015) Simultaneous determination of five naphthoylindole-based synthetic cannabinoids and metabolites and their deposition in human and rat hair. $J$ Pharm Biomed Anal 102:162-175.

Kneisel S, Speck M, Moosmann B, Corneillie TM, Butlin NG, and Auwärter V (2013) LC/ESI-MS/MS method for quantification of 28 synthetic cannabinoids in neat oral fluid and its application to preliminary studies on their detection windows. Anal Bioanal Chem 405:4691-4706.

Lefever TW, Marusich JA, Thomas BF, Barrus DG, Peiper NC, Kevin RC, and Wiley JL (2017) Vaping synthetic cannabinoids: a novel preclinical model of e-cigarette use in mice. Subst Abuse 11:1178221817701739.

Makriyannis and Deng (2000) https://patentimages.storage.googleapis.com/74/6a/cd/ 316c6e25d934d1/WO2001028557A1.pdf

McGregor IS, Issakidis CN, and Prior G (1996) Aversive effects of the synthetic cannabinoid CP 55,940 in rats. Pharmacol Biochem Behav 53:657-664.

Musshoff F, Madea B, Kernbach-Wighton G, Bicker W, Kneisel S, Hutter M, and Auwärter V (2014) Driving under the influence of synthetic cannabinoids ("spice"): a case series. Int J Legal Med 128:59-64.

Ovadia H, Wohlman A, Mechoulam R, and Weidenfeld J (1995) Characterization of the hypothermic effect of the synthetic cannabinoid HU-210 in the rat. Relation to the adrenergic system and endogenous pyrogens. Neuropharmacology 34:175-180.

Patton AL, Chimalakonda KC, Moran CL, McCain KR, Radominska-Pandya A, James LP, Kokes C, and Moran JH (2013) K2 toxicity: fatal case of psychiatric complications following AM2201 exposure. J Forensic Sci 58:1676-1680.

Rawls SM, Cabassa J, Geller EB, and Adler MW (2002) CB1 receptors in the preoptic anterior hypothalamus regulate WIN 55212-2 [(4,5-dihydro-2-methyl-4(4-morpholinylmethyl)-1-(1-naphthalenyl-carbonyl)-6H-pyrrolo[3,2,1ij]quinolin-6-one]induced hypothermia. J Pharmacol Exp Ther 301:963-968.

Schindler CW, Gramling BR, Justinova Z, Thorndike EB, and Baumann MH (2017) Synthetic cannabinoids found in "spice" products alter body temperature and cardiovascular parameters in conscious male rats. Drug Alcohol Depend 179: 387-394.

Smith PB, Compton DR, Welch SP, Razdan RK, Mechoulam R, and Martin BR (1994) The pharmacological activity of anandamide, a putative endogenous cannabinoid, in mice. $J$ Pharmacol Exp Ther 270:219-227.

Sobolevsky T, Prasolov I, and Rodchenkov G (2012) Detection of urinary metabolites of AM-2201 and UR-144, two novel synthetic cannabinoids. Drug Test Anal 4: $745-753$.

Stein EA, Fuller SA, Edgemond WS, and Campbell WB (1996) Physiological and behavioural effects of the endogenous cannabinoid, arachidonylethanolamide (anandamide), in the rat. Br J Pharmacol 119:107-114.

Tuv SS, Krabseth H, Karinen R, Olsen KM, Øiestad EL, and Vindenes V (2014) Prevalence of synthetic cannabinoids in blood samples from Norwegian drivers suspected of impaired driving during a seven weeks period. Accid Anal Prev 62: $26-31$.

World Health Organization (2014) AM-2201 Critical Review Report, Agenda item 4.7, pp 1-30, World Health Organization, Geneva, Switzerland.

Wikström M, Thelander G, Dahlgren M, and Kronstrand R (2013) An accidental fatal intoxication with methoxetamine. J Anal Toxicol 37:43-46.

Yeakel JK and Logan BK (2013) Blood synthetic cannabinoid concentrations in cases of suspected impaired driving. J Anal Toxicol 37:547-551.

Address correspondence to: Dr. Michael H. Baumann, Designer Drug Research Unit (DDRU), Intramural Research Program, NIDA, NIH, 333 Cassell Drive, Suite 4400, Baltimore, MD 21224. E-mail: mbaumann@mail.nih.gov 